Cas clinique

\title{
Sinusalgies maxillaires radiotransparentes par agenesie de l'ostium membraneux
}

\author{
R. Jankowski ${ }^{a}$, C. Rumeau ${ }^{\mathrm{a}, *, \mathrm{~b}}$, D.T. Nguyen ${ }^{\mathrm{a}}$, P. Gallet ${ }^{\mathrm{a}}$ \\ a Service ORL et chirurgie cervico-faciale, hôpitaux de Brabois, centre hospitalier universitaire de Nancy, rue du Morvan, 54511 Vandoeuvre-les-Nancy \\ cedex, France \\ ${ }^{\mathrm{b}}$ EA 3450 DevAH-développement, adaptation et handicap, regulations cardio-respiratoires et de la motricité, université de Lorraine, 9, avenue de la \\ Forêt-de-Haye, 54505 Vandoeuvre-les-Nancy, France
}

\section{N F O A R T I C L E}

\section{Mots clés :}

Sinusite

Sinusalgie

Diagnostic

Scanner

Méatotomie

\begin{abstract}
R É S U M É
Introduction. - L'absence d'opacité scanographique lors du bilan de douleurs faciales conduit classiquement à éliminer une étiologie sinusienne. Nous rapportons une observation qui contredit cet adage et révèle une nouvelle cause de sinusalgie.

Observation. - Un homme de 16 ans présentait un tableau de "sinusites aiguës hyperalgiques " récidivantes déclenchées par les changements de pression (altitude, plongée, surf), sans opacité sinusienne au scanner. Pourtant l'exploration chirurgicale a permis de constater l'absence de tout ostium maxillaire (principal ou accessoire). Une antrostomie chirurgicale a mis fin aux douleurs.

Discussion. - La physiopathologie de ce faux tableau de "sinusites aiguës récidivantes " et l'efficacité de l'antrostomie s'expliquent par la théorie évo-dévo de la formation et du rôle des sinus paranasaux. Ce cas illustre une absence d'abouchement dans l'ethmoïde du sac membranaire tapissant la cavité sinusienne, formée après dégénérescence de la moelle érythropoïétique dans l'os maxillaire. La production permanente de monoxyde d'azote par l'épithélium sinusien est éliminée par simple diffusion transmembranaire en condition environnementale stable, mais insuffisamment lors des changements pressionnels rapides, provoquant des sinusalgies parfois hyperalgiques. Cette observation ouvre la voie à de plus amples études sur le rôle des sinus dans la pathologie faciale et la physiologie respiratoire.

(c) 2018 Elsevier Masson SAS. Tous droits réservés.
\end{abstract}

\section{Introduction}

Les sinus paranasaux, découverts par les anatomistes de la Renaissance, n'ont pu être explorés cliniquement qu'à partir de l'avènement des rayons $X$ découvert par Roentgen à la fin du XIX siècle [1]. Le diagnostic de sinusite repose depuis la fin du XIX siècle sur une modification de leur transparence aux rayons $\mathrm{X}$.

Cet article a pour but d'affiner la place du scanner dans le diagnostic radiologique de sinusite. Nous rapportons en effet un cas de sinusalgies aiguës récidivantes avec un bilan scanographique interprété initialement comme "normal " en l'absence de toute opacité sinusienne. Ces sinusalgies semblaient dues à une absence constitutionnelle de perforation de la membrane sinusienne dans

DOI de l'article original : https://doi.org/10.1016/j.anorl.2018.10.001.

约 Ne pas utiliser pour citation la référence française de cet article mais celle de l'article original paru dans European Annals of Otorhinolaryngology Head and Neck Diseases en utilisant le DOI ci-dessus.

* Auteur correspondant.

Adresse e-mail : c.rumeau@chru-nancy.fr (C. Rumeau). le méat moyen ethmoïdal, et ont été guéries par une antrostomie chirurgicale ayant rétabli l'évacuation du monoxyde d'azote naturellement produit par la muqueuse maxillaire.

\section{Observation}

Un jeune homme de 16 ans consultait suite à une " sinusite aiguë hyperalgique " survenue quelques semaines auparavant lors d'un séjour de ski. Dès son arrivée à $1800 \mathrm{~m}$ d'altitude, il a ressenti une violente douleur au niveau de l'incisive latérale gauche associée à " une sensation de dent mobile qui semblait propulsée en avant ». La douleur diffusait en quelques heures à toute la région sous-orbitaire gauche, l'empêchait de dormir dès la première nuit, et le clouait au lit pendant trois jours malgré une antibiothérapie débutée le lendemain de son arrivée. Elle s'estompait lentement à partir du quatrième jour pour disparaître complètement après son retour à domicile trois jours plus tard. Il ne se souvenait plus s'il y avait eu des signes rhinologiques associés, mais il n'y avait pas eu de mouchage purulent et la dent examinée par son dentiste après son retour était saine. 

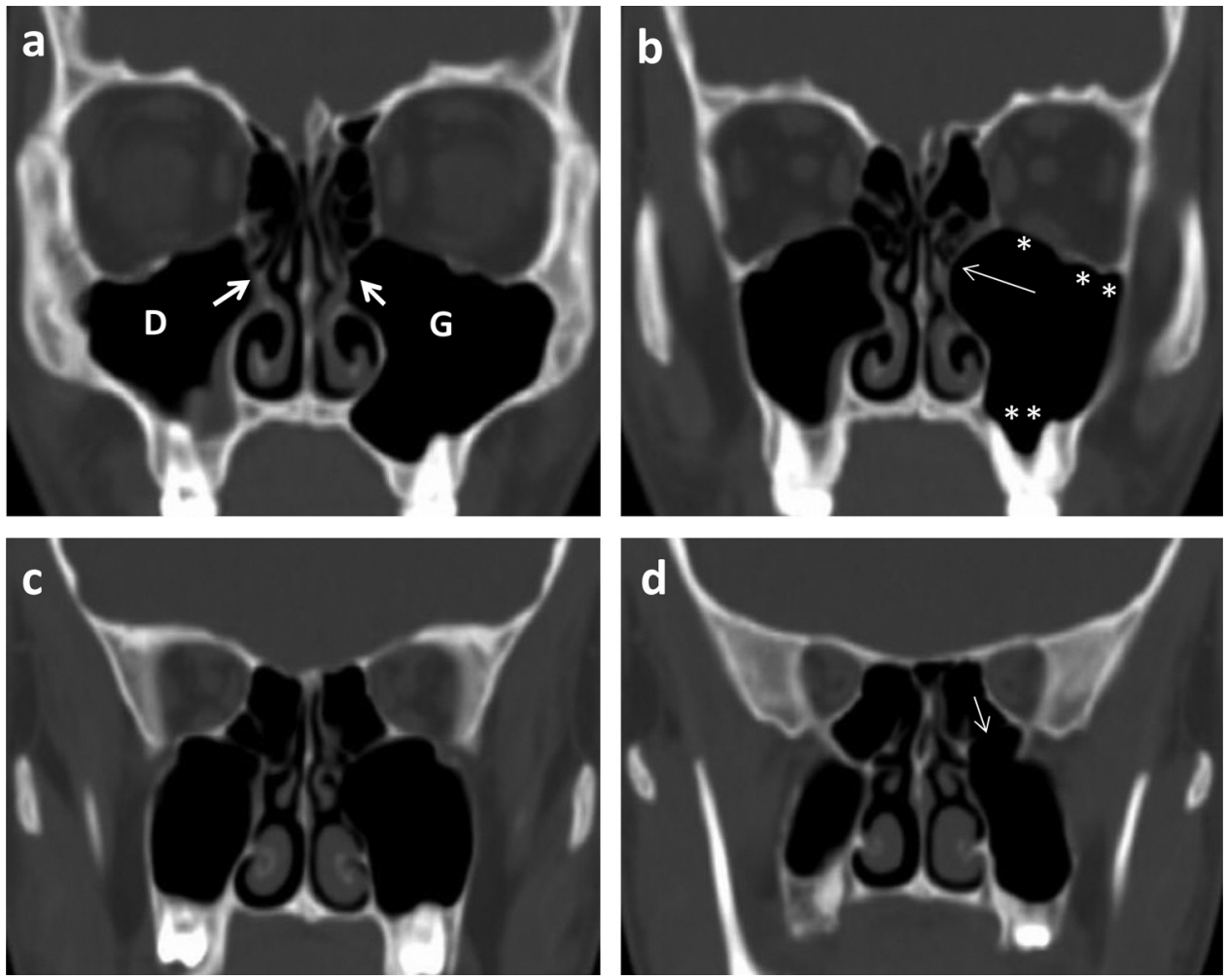

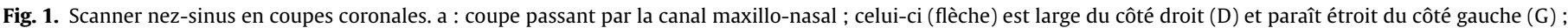

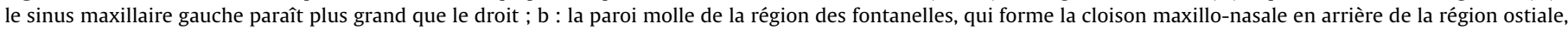

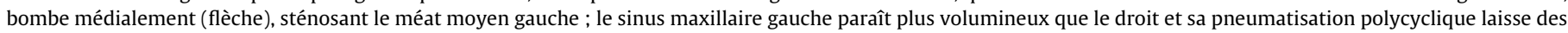

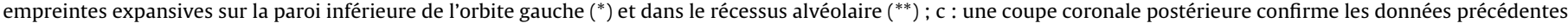

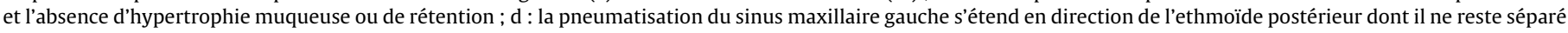
que par la membrane maxillaire (flèche).

Il avait présenté quelques mois auparavant un épisode similaire mais moins douloureux à la suite d'une plongée profonde en apnée lors d'un cours de sauvetage, qui l'avait conduit à interrompre cette formation.

Des douleurs similaires étaient en fait apparues régulièrement deux ans auparavant lors de la pratique du surf, mais elles ne duraient pas plus d'une demi-heure initialement.

Il n'y avait aucun dysfonctionnement nasal chronique entre les crises. La radio-transparence du sinus maxillaire gauche (ainsi que des autres sinus et de l'ethmoïde) était normale, sans aucune hypertrophie muqueuse ou signe de rétention, écartant a priori un barotraumatisme classique.

La morphologie du sinus maxillaire gauche permettait cependant d'évoquer l'hypothèse d'un pneumosinus dilatans (Fig. 1). Le pneumosinus dilatans est une pathologie d'origine osseuse produisant un remodelage des parois sinusiennes lié à une activité ostéoclastique et ostéoblastique. Il a été montré que l'association d'un TEP-scanner au fluorure de sodium (FNa) montrant un hypermétabolisme et d'un Tep au fluoro-désoxy-glucose (FDG) sans hypermétabolisme permettait d'en établir le diagnostic [2]. Chez ce patient, les deux TEP étaient normaux.

L'exploration chirurgicale sous anesthésie générale du méat moyen gauche, après unciformectomie, ne retrouvait pas d'ostium maxillaire, ni principal, ni accessoire. La membrane du sinus maxillaire était alors perforée avec l'aspirateur à l'endroit où l'ostium aurait dû se trouver normalement et une antrostomie de diamètre $7 \mathrm{~mm}$ était créée à la pince coupante. Celle-ci était perméable à la consultation du premier mois postopératoire.

Un an plus tard, le patient disait avoir repris le surf, la plongée et le ski alpin sans plus aucune douleur.

\section{Discussion}

L'origine sinusienne des crises douloureuses chez ce patient était a posteriori confirmée par leur disparition suite à l'antrostomie. L'absence d'ostium maxillaire gauche constatée sous anesthésie générale était pour autant difficile à prévoir sur le scanner (Fig. 1).

La pneumatisation du sinus maxillaire gauche ne s'accompagnait ni d'anomalie muqueuse ni d'opacité rétentionnelle, mais paraissait plus importante qu'à droite, laissant des empreintes d'allure expansive sur la paroi inférieure de l'orbite et dans le récessus alvéolaire. L'hypothèse d'un pneumosinus dilatans maxillaire gauche était évoquée. Le TEP-scanner au FDG explorant la muqueuse et le TEP-scanner au FNa explorant l'os avaient été réalisés dans le but de confirmer le diagnostic de pneumosinus dilatans et d'écarter celui d'une hyperpneumatisation banale [2]. L'absence de fixation de l'os au TEP-FNa mettait en doute l'hypothèse du pneumosinus dilatans, mais l'efficacité de l'antrostomie dans le traitement du pneumosinsus dilatans incitait néanmoins à l'intervention chirurgicale [2].

L'exploration chirurgicale sous anesthésie générale (Fig. 2a) débutait par une unciformectomie du segment horizontal permettant d'exposer la région de l'ostium (Fig. 2b). Aucun ostium ni ouvert, ni fermé ne pouvait être identifié (Fig. 2c,d) [3]. La palpation avec l'aspirateur de la région ostiale entraînait la formation de bulles gazeuses à la surface de la membrane sinusienne (Fig. 2e). Celle-ci était simplement perforée avec une pince, créant une ouverture limitée, d'environ $7 \mathrm{~mm}$ (Fig. 2f), non-élargie par la suite par une méatotomie moyenne académique étendue à la région des fontanelles. 

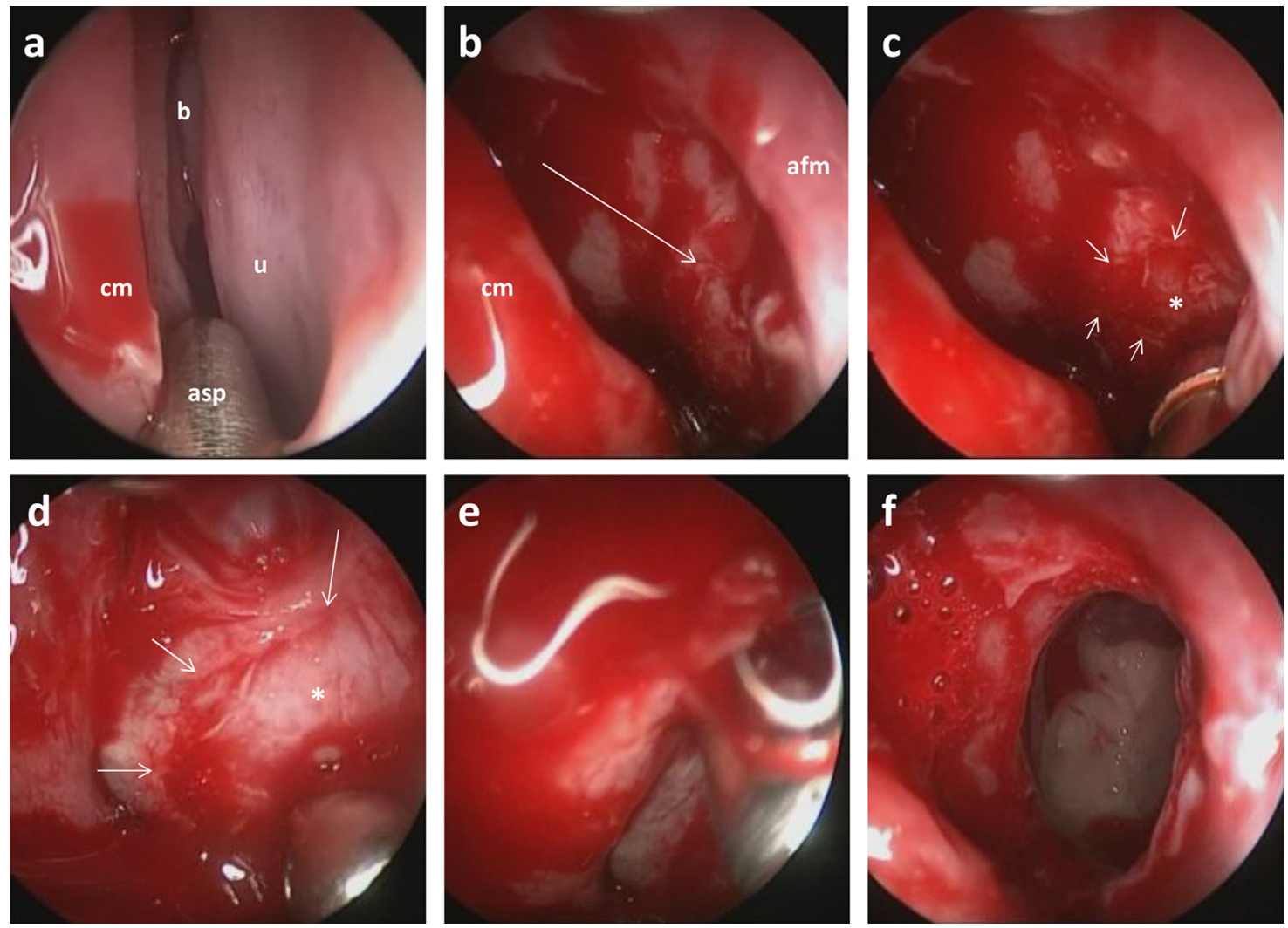

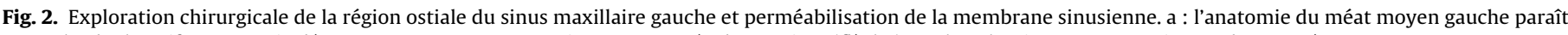

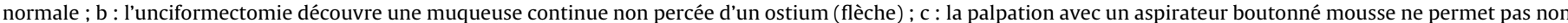

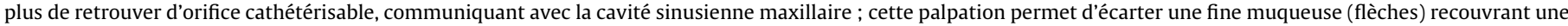

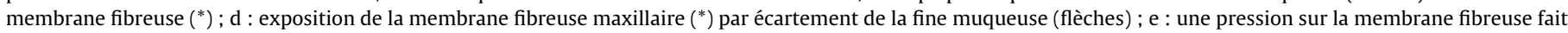

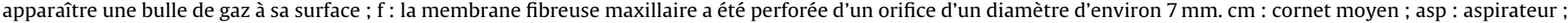
$\mathrm{u}$ : unciforme ; b : bulle ; afin : apophyse frontale du maxillaire.

La compréhension de l'efficacité de l'antrostomie et de la physiopathologie de ce faux tableau de "sinusites aiguës récidivantes", plus exactement de sinusalgies aiguës récidivantes radiotranparentes, peut en fait s'appuyer sur la théorie évo-dévo des sinus. Ces derniers ne seraient pas formés à partir des cellules ethmoïdales mais résulteraient d'une désintégration de la moelle osseuse des os maxillaires, frontaux et sphénoïdaux aboutissant à des cavités tapissées d'un épithélium producteur de NO [4]. La fonction sphinctérienne de l'ostium [3] régulerait la libération de ce gaz sous forme de bolus [5]. Outre toutes les hypothèses avancées à ce jour [6], la vraie raison d'être des sinus semble être leur fonction respiratoire de production, stockage et libération sur commande du monoxyde d'azote (NO), un messager aérocrine qui augmente à la demande l'oxygénation du sang artériel au niveau des alvéoles pulmonaires [7]. La constatation d'une absence possible d'ostium maxillaire semble être un argument supplémentaire en faveur de la théorie évo-dévo de formation des sinus. Comment expliquer sinon que le sinus maxillaire ait pu chez ce patient être pneumatisé à partir d'une cellule ethmoïdale alors même qu'aucune solution de continuité n'existait entre les deux structures ? Chez ce patient, la régression de la moelle osseuse créant la cavité sinusienne tapissée de sa membrane propre n'aurait pas abouti à son abouchement dans l'ethmoïde. Le percement chirurgical de cette membrane n'a certainement pas recréé la fonction sphinctérienne, mais le NO, qui en conditions de pression atmosphérique stable semblait pouvoir diffuser à travers la membrane pour être évacué (Fig. 2e), peut à présent être évacué par cet orifice artificiel (Fig. 2f) en cas de variations pressionnelles brutales (altitude, plongée, surf), mettant ainsi fin aux douleurs.
Le rôle du dysfonctionnement des ostiums sinusiens dans les douleurs faciales mériterait à présent d'être pris en compte et évalué à la lumière de cette observation. Ainsi une agénésie de l'ostium membraneux mériterait d'être recherchée systématiquement dans les barotraumatismes classiques avec opacité sinusienne. Le cas clinique présenté ici correspond peut-être à une étiologie rare de douleurs faciales, mais qui peut être évoqué devant un tableau de douleurs "pressionnelles ». L'absence totale de toute opacité dans un sinus fermé n'exclut pas une origine sinusienne des douleurs et interroge de plus les concepts traditionnels concernant la " ventilation » et le « drainage » des sinus paranasaux.

\section{Conclusion}

Cette observation illustre la théorie évo-dévo de pneumatisation des sinus paranasaux. Elle permet également d'affiner l'interprétation des images du scanner nez-sinus dans le diagnostic des dysfonctionnements naso-sinusiens douloureux. Elle ouvre enfin la voie à de plus amples études sur le rôle des sinus dans la pathologie faciale et dans la physiologie respiratoire.

\section{Source de financement}

Aucun.

\section{Déclaration de liens d'intérêts}

Les auteurs déclarent ne pas avoir de liens d'intérêts. 


\section{Références}

[1] Willemot J. Naissance de l'oto-rhino-laryngologie belge. In: Willemot J, Ed. Naissance et développement de l'oto-rhino-laryngologie dans l'histoire de la médecine. B-ENT 1981;35:1-1698.

[2] Jankowski R, Kuntzler S, Boulanger N, et al. Is pneumosinus dilatans an osteogenic disease that mimics the formation of a paranasal sinus? Surg Radiol Anat 2014;36:429-37.

[3] Jankowski R, Rumeau C. Physiology of the paranasal sinus ostia: endoscopic findings. Eur Ann Otorhinolaryngol Head Neck Dis 2018;135:147-8.

[4] Jankowski R. The evo-devo origin of the nose anterior skull base and midface. Paris: Springer; 2013, ISBN 978-2-8178-0421-7.
[5] Maniscalco M, Weitzberg E, Sundberg J, et al. Assessment of nasal and sinus nitric oxide output using single-breath humming exhalations. Eur Respir J 2003;22:323-9.

[6] Marquez S. The paranasal sinuses: the last frontier in craniofacial biology. Anatom Rec 2008;261:1350-61.

[7] Lundberg J, Lundberg J, Settergreen G, et al. Nitric oxide, produced in the upper airways, may act in an "aerocrine" fashion to enhance pulmonary oxygen uptake in humans. Acta Physiol Scand 1995;155: 467-8. 